Revista de Negócios_ISSN 1980.4431_vol. 19, n. 3, p. 23_40, 2014_DOI:10.7867/19804431.2014v19n3p23_40

\title{
A percepção de gestores da construção civil acerca do conceito de estratégia organizacional: Um estudo exploratório
}

\section{The managers of building perceptions about the concept of organizational strategy: An exploratory study}

\author{
Maurício Caetano Nedeff \\ Universidade de Caxias do Sul - Brasil \\ mcnedeff@netprata.com.br \\ Mikael Dalberto \\ Universidade de Caxias do Sul - Brasil \\ mldalberto@yahoo.com.br \\ Deonir De Toni \\ Universidade de Caxias do Sul - Brasil \\ deonirdt@terra.com.br \\ Gabriel Sperandio Milan \\ Universidade de Caxias do Sul - Brasil \\ gsmilan@ucs.br \\ Paulo Fernando Pinto Barcellos \\ Universidade de Caxias do Sul - Brasil \\ pfpbarce@ucs.br
}

Recebido em 8 de janeiro de 2013. Alterado em 25 de agosto de 2014. Aprovado em 9 de setembro de 2014.

Editor Responsável: Edson Roberto Scharf, Dr.

Processo de avaliação por double blind review

\section{Resumo}

A Estratégia Organizacional para as empresas garante direcionamento e posicionamento estratégico no contexto atual. No entanto, a compreensão do termo, seu significado, necessita de maior reflexão sobre sua definição para suprimir dúvidas a partir da visão dos gestores. Com isso, o objetivo é identificar a imagem de estratégia organizacional a partir da visão de gestores de empresas ligadas à construção civil, em uma cidade da Serra Gaúcha. Com relação ao método de pesquisa, foi empregada a Técnica de Configuração de Imagens Ideativas (TCID), que é uma variação do MCI (Método de Configuração de Imagem); onde o MCI consiste em uma técnica de configuração de imagem de objetos mais "tangíveis" e, o TCID uma técnica de configuração de imagem de objetos "intangíveis", puramente ideativos (DE TONI et al., 2006). Foram realizadas vinte entrevistas com os gestores, em novembro e dezembro de 2010. Os resultados deste estudo apresentaram 32 atributos, que foram distribuídos dentre as cinco dimensões de estratégia, propostas por Mintzberg (1987), os Cinco Ps - plano, pretexto, padrão, posição e perspectiva. O estudo possibilita a observação da imagem que os 
entrevistados, gestores de empresas, têm em relação ao conceito de Estratégia Organizacional. Os resultados identificaram que para os gestores pesquisados o conceito de estratégia está mais ligado às dimensões Plano e Perspectiva. Dentre as contribuições deste artigo pode-se destacar a proposição de um método que auxilia a melhor entender a configuração de um conceito, neste caso, de Estratégia Organizacional.

Palavras-chave: Estratégia Organizacional; Método de Configuração de Imagem (MCI); Técnica de Configuração de Imagens Ideativas (TCID); Construção Civil.

\begin{abstract}
Organizational Strategy for companies ensures targeting and positioning strategic in the current context. However, understanding of the term, its meaning, needs further reflection on its setting to suppress doubts from the perspective of the managers. Thus, the aim of this work is to identify the image of organizational strategy from the perspective of managers linked to building, in a city of Serra Gaucha. With regard to the research method, was employed the Technical of Configuration Ideational Images (TCID), which is a variation of the ICM (Image Configuration Method); where ICM consists of a configuration technique of objects' image more "tangible" and the TCID technique configuration of objects' image "intangible", purely ideational (DE TONI et al., 2006). Twenty interviews were conducted with managers, in November and December of 2010. Results of this study showed 32 attributes, which were distributed among five dimensions of strategy, proposed by Mintzberg (1987), the Five Ps - plan, pretext, pattern, position and perspective. The study allows the observation image that respondents, company managers, have in relation to the concept of Organizational Strategy. Results of this study identified that the management searched for the term strategy is more related to the dimensions Plan and Perspective. Among contributions of this paper can be highlighted of propose a method that helps to better understand the configuration of a concept, in this case, the Organizational Strategy.
\end{abstract}

Keywords: Organizational Strategy; Image Configuration Method (ICM); Technical of Configuration Ideational Images (TCID); Building.

\section{Introdução}

Parte do sucesso ou fracasso de uma organização está fortemente relacionado ao direcionamento estratégico que as organizações buscam. Ao considerar a relevância do desenvolvimento e posterior utilização da estratégia organizacional para o desenvolvimento das empresas, é importante entender o significado que os gestores dão para o conceito de estratégia organizacional, termo frequentemente empregado no cotidiano das empresas. Há, porém, uma carência de reflexão sobre a sua definição e melhores desdobramentos, o que pode contribuir para tornar o conceito difuso e impreciso para grande parte dos gestores (FONSECA; MACHADO-DA-SILVA, 2001).

Dessa forma este trabalho tem o objetivo de identificar a imagem coletiva que alguns gestores de empresas da região da Serra Gaúcha tem em relação a estratégia organizacional, de modo a categorizar os atributos dessa imagem com base nas cinco dimensões de estratégia propostas por Mintzberg (1987), os cinco Ps: plano, pretexto, padrão, posição e perspectiva.

Assim, para identificar a imagem que o conceito de estratégia organizacional tem para os empresários do segmento da construção civil foi utilizado a Técnica de Configuração de Imagens Ideativas (TCID). Está é uma adaptação do Método de Configuração de Imagem (MCI) desenvolvida e evoluida por Schuler (2004), e De Toni (2005) e Schuler, De Toni e Milan (2014), é a técnica de configuração de imagem de servicos (TCIS), implementada por De Toni, Milan e Schuler (2005) e Milan e De Toni (2008). Portanto, o pressuposto básico da TCID é a identificação de como os conceitos podem ser configurados pelo público alvo de interesse.

Para a aplicação desta técnica foram entrevistados vinte gestores, os quais estão ligados a empresas de construção civil. Os resultados apontam que para os entrevistados o conceito de estratégia organizacional está ligado quase que exclusivamente a ideia de Plano e Pretexto. Dessa forma, dentre as 
contribuições deste artigo pode-se destacar a proposição de um método que auxilia a melhor compreensão da configuração de um conceito, neste caso, de estratégia organizacional. Deste modo, este trabalho está estruturado em quatro seções. A primeira, apresenta a revisão da literatura. Na segunda seção, o método de pesquisa é exposto. Na terceira, é abordada a análise dos resultados, onde consta o Gráfico da Configuração da Imagem (GCI). E, na quarta seção, são tecidas as conclusões e considerações finais.

\section{Revisão da literatura}

O conceito de estratégia organizacional pode ser explorado de diferentes perspectivas, no caso deste artigo ele segue o direcionamento de Mintzberg (1987). Para entender este conceito também é apresentado como as imagens estão organizadas na memória e como podem impactar no pensamento estratégico.

\subsection{Estratégia organizacional e suas dimensões - Os Cinco Ps}

Estratégia organizacional pode ser compreendida como a busca por uma posição competitiva favorável, caracterizada pela criação de uma posição exclusiva e valiosa, de modo a efetuar tradeoffs claros, contra as demais forças competitivas existentes no mercado, responsáveis pela rivalidade e a competição (PORTER, 2001; 2004). Portanto, é recomendável o fortalecimento da cadeia de valor do negócio, maximizar a performance dos processos e atividades da empresa por meio da integração da cadeia de suprimentos em torno dos objetivos comuns e imprescindíveis para o seu sucesso (PORTER, 2004).

Segundo Eisenhardt (1999), a estratégia é um processo de tomada de decisão da empresa, reforçado pela sua intuição coletiva. Por sua vez, Quinn
(1980) afirma que a estratégia é o padrão ou o plano que integra objetivos e metas, políticas e uma sequência de ações coerentes com a estrutura e os recursos da empresa. Para Porter (1996) como a estratégia se baseia em atividades únicas, singulares a uma determinada empresa, a definição de estratégia está relacionada a ser diferente dos competidores, significa escolher um conjunto de atividades diferenciadas para possibilitar a geração e a entrega de um mix único de valor aos clientes. Na visão de Daft (1995), a estratégia organizacional pode ser definida como um plano de interação com os ambientes competitivos, de modo a alcançar os objetivos da organização. Thompson Jr. e Strickland III (2004) também comentam que a estratégia consiste em um conjunto de mudanças competitivas e abordagens comerciais focadas na melhoria do desempenho organizacional.

Ao considerar a complexidade intrínseca ao conceito de estratégia, Mintzberg (1987) e Mintzberg et al. (2006) defendem o reconhecimento explícito das definições múltiplas que a mesma compreende sem a necessidade de uma "definição correta". Nesta direção, Mintzberg (1987) apresenta cinco dimensões de definições - plano, pretexto, padrão, posição e perspectiva, sobre as quais existem, logicamente, algumas interrelações:

a) Plano (plan): é possível dizer que a estratégia é um conjunto de diretrizes para guiar determinada situação. Neste caso, a estratégia é elaborada previamente à ação e, na maioria dos casos, é explicita, e apresenta um planejamento formal. Pode-se considerar que a estratégia seja um plano formal e específico para as escolhas de uma organização em um cenário, são intenções 
que asseguram o alcance dos objetivos vislumbrados;

b) Pretexto (pretext): dimensão da estratégia que constitui uma espécie de manobra ou truque, com a intenção de obter uma vantagem sobre um competidor ou um concorrente. Por exemplo, uma empresa pode divulgar no mercado a notícia de expansão de sua unidade fabril apenas com o objetivo de desencorajar seu concorrente a montar uma unidade em tal local. Esta manobra concentra atenção nos aspectos dinâmicos, competitivos e nos sinais do mercado;

c) Padrão (pattern): característica de comportamento padrão de uma organização que pode ser observado e percebido pelo mercado, ou seja, um padrão de uma corrente de ações. Quando Picasso pintou quadros azuis por um período, assim como foi estratégia o comportamento da Ford Motors Company quando Henry Ford oferecia o modelo $\mathrm{T}$ na cor preta. Em outras palavras, esta definição de estratégia se refere a consistência no comportamento, pretendida ou não;

d) Posição (position): indica a demarcação do campo de atuação da empresa como nicho ou a posição que a empresa ocupa no mercado para evitar os competidores ou se sobressair em relação a eles. Trata-se de uma forma de contextualizar a empresa, inserido-a em um ambiente competitivo, é a tática para detalhar cenários, mais ou menos importantes em decorrência dos ambientes;

e) Perspectiva (perspective): a estratégia é para a organização aquilo que a personalidade é para um indivíduo. Trata-se de estratégia como um conceito, consiste não apenas em uma posição escolhida ou desejada ao longo do tempo, mas também na forma que os gestores enxergam as oportunidades para a empresa perante o mercado. Para atender às perspectivas, a empresa pode adquirir uma posição agressiva com a aquisição de novas tecnologias e prospecção de novos mercados, bem como pode adquirir uma posição defensiva, ao buscar criar barreiras de proteção em relação a concorrentes e ameaças.

As cinco dimensões da estratégia apresentadas se integram, se relacionam sinergicamente e se complementam. Juntas além de oferecer uma base estratégica, também potencializam o desenvolvimento do processo de exercício de reflexão estratégica. Assim, por meio de planejamento, é possível antecipar o futuro pretendido e a ação integrada dentro da organização, com foco no objetivo a ser alcançado, ser capaz de posicionar a organização em termos de volume de negócios, participação de mercado (market share), lucro e rentabilidade (MINTZBERG, 1987; MINTZBERG; AHLSTRAND; LAMPEL, 2010; MINTZBERG, 2004; MINTZBERG et al., 2006).

\subsection{Imagem e sua influência no comportamento dos estrategistas}

A imagem possui papel importante sobre a estratégia, pois de acordo com Neves (2003), a imagem é como percebemos a realidade. A imagem do indivíduo na sociedade depende da interpretação e apreciação de sua imagem pela sociedade. Portanto, cada um tem a 
liberdade para perceber a realidade do jeito que lhe aprouver, logo as alternativas imaginativas são infinitas, à vista disso, para Boulding (1968), a imagem provém de uma estrutura subjetiva de conhecimentos, é resultado de toda a experiência passada do sujeito, em alguma forma de contato com o objeto da imagem. Assim, as imagens possuem um papel fundamental no direcionamento estratégico das organizações. Por meio das imagens, é possível moldar o pensamento e direcionar as ações estratégicas das organizações (MACHADO-DA-SILVA; FONSECA; FERNANDES, 2000).

Como modelos mentais, as imagens são aprendizagens passadas que se tornam a base para a interpretação da realidade, influenciam fortemente as ações das pessoas pelo viés cognitivo. Por meio destes modelos mentais, o processo de tomada de decisão-é baseado na analogia das realidades vivenciadas pelo indivíduo, de modo a evitar, assim, o contato direto com o desconhecido, são as inferências, ao qual o indivíduo pressupõe a probabilidade de ocorrer um fato com base uma proporção de modelos de eventos anteriores, deste modo ao longo do processo de desenvolvimento, o indivíduo deixa de utilizar marcas externas e passa a se apropriar de representações mentais do mundo real, os signos internos (OLIVEIRA, M.; 1995). As imagens constituem uma das mais importantes partes do intelecto humano, capaz de influenciar e direcionar o comportamento das pessoas (SCHULER; DE TONI; MILAN, 2014) Por isso, resultados de sucesso, ou de fracasso, são subsídios para o direcionamento de novas decisões e novas estratégias (MACHADO-DASILVA; FONSECA; FERNANDES, 2000; GENTNER, 1983).

Imagens adquiridas ao longo do tempo por um determinado indivíduo se transformam em uma base de referências para a compreensão das várias situações e ambientes vivenciados. A imagem atual gera um ponto de partida para novas análises, determina quais situações receberão mais ou menos atenção, bem como expressam como as pessoas vêem ou representam determinado objeto. Portanto, imagens fortemente constituídas na mente de um gestor podem fazer com que o mesmo não observe as mudanças ambientais que estejam ocorrendo ao seu redor, relevantes ao processo de tomada de decisões. Desta forma, é possível identificar que a imagem influencia diretamente na observação das mudanças do ambiente e no processamento das informações que os gestores recebem sobre o contexto, impactam no seu comportamento, nas suas atitudes e nas suas ações (ZALTMAN, 1997; SENGE, 2012; MACHADO-DA-SILVA; FONSECA; FERNANDES, 2000).

\subsection{Organização das imagens na memória}

Observar as diferentes perspectivas do entendimento de como as imagens são organizadas na mente do indivíduo, duas teorias se destacam: a Teoria das Representações Sociais e a Teoria do Núcleo Central.

A Teoria das Representações Sociais (TRS), abordada inicialmente por Moscovici $(1978,1988)$, busca explicar a forma pela qual ocorrem as influências sociais e como cada indivíduo constitui e armazena seus modelos mentais. Tal teoria apresenta a constituição dos modelos mentais como um processo socialmente mediado, é o modo como um grupo de pessoas forma o conhecimento do cotidiano que influencia no comportamento das pessoas e do contexto organizacional. Para Breakwell (2001), os canais de informação apresentam forte influência na formação da imagem dos indivíduos, são as ligações sociais compreendidas por um conjunto de crenças, convicções e valores amplamente compartilhados pelas pessoas, com a 
finalidade principal de familiarizar os eventos e seus significados que foram atribuídos pela sociedade.

A TRS sugere a determinação dos modelos mentais de qualquer objeto, ou fenômeno, por meio da inclinação do indivíduo, que constitui, uma forma de ancoragem mental. Este processo observa os significados difundidos socialmente e o consenso por meio da comunicação entre as pessoas (BREAKWELL, 2001). Deste modo, é possível perceber forte influência das relações social nos modelos mentais, bem como nos modelos de estratégia organizacional, a partir dos conceitos e das crenças compartilhadas entre os membros de uma subcultura discreta, específica, particular. Isto não significa que os membros de uma mesma subcultura apresentem um modelo mental idêntico de estratégia organizacional, mas que podem compartilhar uma série de elementos (MILAN; DE TONI, 2008).

A Teoria do Núcleo Central sugere que o homem processa e organiza as informações de forma dinâmica, adapta as interpretações ao meio ambiente ao qual está inserido e permanece em constante mutação, de forma evolutiva em torno de um Núcleo Central e de um conjunto de elementos periféricos. Portanto, os atributos dos modelos mentais serão tão mais próximos de uma imagem coletiva quanto mais elevado for o número de indivíduos que os perceberem da mesma maneira. (ABRIC, 1984; SÁ, 1996; 2002; CHAPMAN; FERFOLJA, 2001). Os atributos são compreendidos como rede de relações entre os dados presentes na mente humana, são expectativas que fornecem uma estrutura capaz de interpretar e compreender as informações sobre determinado objeto que chegam ao sujeito (AKHTER; ANDREWS; DURVASULA, 1994). Logo, o Núcleo Central é determinado em parte pelo modelo do objeto e, em parte, pela relação que o indivíduo mantém com este objeto. Sendo assim, o Núcleo Central é constituído de um conjunto de representações, compostas de um ou mais elementos, onde a sua ausência desestruturaria a percepção do indivíduo, fato que daria uma significação complemente diferente (SÁ, 2002).

Os atributos que compõem o Núcleo Central são marcados pela memória coletiva e são resistentes às mudanças, com objetivo principal de gerar uma imagem do contexto. Este sistema central é envolvido por sistemas periféricos flexíveis, sensíveis e adaptáveis ao contexto que está inserido, de modo a permitir a adaptação das diferentes realidades, bem como a diferenciação do conteúdo e a proteção do sistema central. Os sistemas periféricos, são caracterizados pela proximidade às práticas do dia-a-dia e pela capacidade de mudança (ABRIC, 1996; DE TONI, 2005). Desta forma, é possível entender que a imagem é um conjunto de significados e esquemas associados e organizados em torno dos elementos centrais socialmente compartilhados e aceitos em uma subcultura. A partir do uso da imagem mental que os gestores possuem em torno da estratégia organizacional para explicar conceitualmente como se dá a configuração da realidade organizacional, de modo a compreender melhor a estratégia e as práticas organizacionais adotadas, a população e amostra pesquisada (MILAN; DE TONI, 2008).

\section{Metodologia}

O método de pesquisa é constituído pela proposição da Técnica de Configuração de Imagens Ideativas (TCID). A partir desta técnica que tem influência marcante na Teoria das Representações Sociais e na Teoria do Núcleo Central. Deste modo, foi possível entender como uma imagem pode ser configurada na mente dos entrevistados. A seguir será abordado como está técnica foi aplicada, o instrumento de coleta $\mathrm{e}$ tratamento dos dados. 


\subsection{A técnica de Configuração de Imagens Ideativas (TCID)}

A Técnica de Configuração de Imagens Ideativas (TCID) é um instrumento de pesquisa que utiliza por base métodos já desenvolvidos e validados, tais como: o Customer Window (APDG, 1994) e o Framework for Marketing Image Management (BARICH; KOTLER, 1991), além de métodos desenvolvidos por pesquisadores europeus, com o objetivo de acessar a configuração das representações sociais (ABRIC, 1984; MINAYO, 1997; MOSCOVICI, 1978; 2000; SÁ, 2002). O desenvolvimento do MCI é resultado de anos de pesquisa acadêmica por parte de pesquisadores brasileiros (SCHULER, 2004; DE TONI, 2005; MILAN; DE TONI, 2008; SCHULER; DE TONI; MILAN, 2014). Técnica que detecta áreas de problemas e oportunidades, como também contribui para a definição do foco para as gestões futuras da imagem das organizações (SCHULER; DE TONI; MILAN, 2014).

Neste trabalho, se utiliza uma técnica específica, derivada deste método, que é direcionada à configuração de Imagens Ideativas (DE TONI et al., 2006), ou seja, idéias, conceitos e abstrações. Então, com base em uma abordagem predominantemente exploratória, o estudo apresenta os seguintes objetivos: (i) identificar os atributos salientes da imagem de estratégia organizacional, sob o ponto de vista dos entrevistados (gestores de empresas de Nova Prata - Serra Gaúcha); (ii) apontar a Imagem Central e a Periférica do conceito em estudo (estratégia organizacional); (iii) classificar os atributos identificados dentre as cinco dimensões propostas por Mintzberg (1987), os Cinco Ps da estratégia; e (iv) apresentar uma disposição gráfica dos resultados obtidos, disposição esta tangibilizada pelo Gráfico de Configuração da Imagem (GCI), que permite a visualização da configuração da imagem de estratégia organizacional, na perspectiva dos gestores entrevistados.

\subsection{Contexto geral da população da pesquisa e amostra}

Ao considerar o déficit habitacional no Brasil como um grande problema social, os autores optaram por desenvolver a pesquisa em empresas, com o foco nos e gestores que estejam envolvidos diretamente na gerência das seguintes empresas: construtoras, urbanizadoras, imobiliárias e lojas de material de construção. A opção por realizar a pesquisa no município de Nova Prata, na Serra Gaúcha, se deu pelo entendimento da representatividade como centro de uma micro-região, que abrange outros oito municípios, com uma população total de cerca de 50.000 habitantes (IBGE, 2010), com a ressalva de que a amostra é nãoprobabilística, ou seja, é estabelecida por conveniência (MALHOTRA, 2009).

Conforme é apresentado na Tabela 1 foram entrevistados 20 empreendedores sendo que destes, oito são do setor imobiliário, quatro de construtoras, duas urbanizadoras e seis empresários de lojas de materiais de construção.

Tabela 1: Descrição da amostra

\begin{tabular}{c|c|c|c|c}
\hline \multirow{2}{*}{ Resultados } & \multicolumn{4}{|c}{ Tipos de Empresa que Compõem a Amostra } \\
\cline { 2 - 5 } & Imobiliárias & Construtoras & Urbanizadoras & Lojas de Material de Construção \\
\hline Frequência & 8 & 4 & 2 & 6 \\
\hline$\%$ & 40 & 20 & 10 & 30 \\
\hline
\end{tabular}

Fonte: Dados provenientes da pesquisa. 
A percepção de gestores da construção civil acerca do conceito de estratégia organizacional: Um estudo exploratório

O critério utilizado para a definição desta amostra foi a saturação dos dados, visto que a partir das décima quinta entrevista as informações começaram a se repetir, dessa forma, optou-se por finalizar a coleta.

\subsection{O instrumento de coleta de dados e a condução das entrevistas}

Objetivando substituir as perguntas diretas e simples, por um conjunto de estímulos que são capazes de revelar um pouco mais o conteúdo das imagens mentais dos respondentes, foi utilizado um "Roteiro Básico de Questões", através de entrevistas semi-estruturadas (RIBEIRO; MILAN, 2004), com a intenção de estimular a espontaneidade nas respostas, estas pertencentes a algumas categorias de elementos que, na teoria, compõem as imagens na perspectiva dos entrevistados (DE TONI et al., 2006).

A utilização de um roteiro, apresentado na Figura 1, compreendido como um $\Theta$ instrumento mais completo, ao se comparar com o simples questionamento dos entrevistados sobre suas percepções e convicções. Inclusive, Gentner (2001) destaca que somente perguntas diretas não são suficientes, é necessário considerar que as pessoas, normalmente, não articulam plenamente seus conhecimentos, opiniões e ideias quando questionadas desta maneira.

Da mesma forma, ao se observar a natureza global ou holística da imagem, modelos para mensuração com escalas fechadas, como por exemplo, hierarquias, ordens de importância e lista de apelos, necessitariam sofrer complementação com outras abordagens, pois estas técnicas captam apenas fatos específicos e não redes de significados, impressões e representações (DICHTER, 1985).

Sendo assim, o Roteiro Básico de Questões (De TONI et al., 2006) é apresentado conforme a Figura 1, que segue:

Figura 1: Roteiro Básico de Questões

\begin{tabular}{|l|c|}
\multicolumn{1}{|c|}{ Roteiro Básico de Questões } & $\begin{array}{c}\text { Tipo de } \\
\text { Evocação }\end{array}$ \\
\hline 1. Quando eu digo Estratégia Organizacional, qual é a primeira coisa que lhe vem à mente? & Top of Mind \\
\hline 2. Que outras idéias lhe vêm à mente sobre Estratégia Organizacional? & Geral \\
\hline 3. Como você descreve a Estratégia Organizacional? & Cognitiva \\
\hline 4. Qual o significado de Estratégia Organizacional na sua empresa? & Simbólica \\
\hline 5. Quais os sentimentos que lhe vêm à mente quando eu digo Estratégia Organizacional? & Emocional \\
\hline 6. Quais os benefícios que a Estratégia Organizacional traz para a sua empresa? & Funcional \\
\hline
\end{tabular}

Fonte: Adaptado de De Toni et al. (2006).

Relativo às questões utilizadas, De Toni et al. (2006) colocam que as de número “1” e "2"

auxiliam na identificação dos atributos mais prontamente evocados, a partir da memória do entrevistado, quando o objeto de pesquisa é citado, no caso, "Estratégia Organizacional". Estas duas questões buscam verificar $\mathrm{o}$ tom geral da representação mental que o entrevistado tem sobre o conceito. Em relação às questões "3 à 6", tais questões buscam dar uma maior consistência à coleta de dados ao repetir o mesmo questionamento básico por meio de outras perspectivas (abordagens cognitiva, emocional e funcional), que formam, assim, uma escala unidimensional, que se interessa em propor ao entrevistado um maior número de oportunidades de revelar os conceitos que 
fazem parte da configuração da sua imagem.

A partir do "Roteiro Básico de Questões", apresentado na Figura 1, as entrevistas foram implementadas, aplicadas individualmente e em sessão única. A duração média de cada entrevista ficou em cerca de 25 minutos. A condução das entrevistas e o processo de coleta de dados ocorreu durante os meses de novembro e dezembro de 2010.

\subsection{Tratamento dos dados}

Os dados obtidos foram tratados em três etapas, conforme proposto por De Toni et al. (2006). $\mathrm{Na}$ primeira etapa do tratamento dos dados, foi realizada a análise de conteúdo das respostas, através dos pressupostos informados por Bardin (2011). Após a análise de conteúdo, os atributos mencionados pelos entrevistados foram listados, de modo a gerar, um conjunto dos atributos que formam a imagem de estratégia organizacional para o conjunto de entrevistados.

$\mathrm{Na}$ execução da segunda etapa, foram obtidas a frequência e a ordem de evocação dos atributos, de acordo com os pressupostos de Abric (1984) e Vergès (1992). Nesta etapa, o objetivo era criar uma distinção entre os atributos mais próximos e os mais distantes em relação ao termo indutor empregado, que, neste trabalho, é a "Estratégia Organizacional".

É importante comentar que os atributos mais próximos são pertencentes à Imagem Central e os atributos mais distantes à Periferia da Imagem. Valores de Frequência (VF) e Valores de Ordem (VO) foram atribuídos para cada atributo identificado. Para a obtenção do VO, foi adotado o critério apresentado na Figura 2. Para a obtenção do Valor Total (VT) de cada atributo, foi realizada a soma simples entre VF e VO.

Figura 2: Critérios para o cálculo dos Valores de Ordem (VO) de citação

\begin{tabular}{|c|c|}
\hline Ordem de Citação & Valor (Pontos) Atribuídos \\
\hline Atributo citado em $1^{\circ}$ lugar & 5 pontos \\
\hline Atributo citado em $2^{\circ}$ lugar & 4 pontos \\
\hline Atributo citado em $3^{\circ}$ lugar & 3 pontos \\
\hline Atributo citado em $4^{\circ}$ lugar & 2 pontos \\
\hline Atributo citado em $5^{\circ}$ lugar & 1 pontos \\
\hline Atributo citado em $6^{\circ}$ lugar & sem pontuação \\
\hline
\end{tabular}

Fonte: Adaptado de De Toni et al. (2006).

Com o intuito de atribuir as zonas de proximidade com o termo indutor, foi utilizado como critério discriminatório o VT dos atributos. Então, a partir do VT, foi aplicado o procedimento de "Divisão de Quartis". Por meio desta divisão foram criados quatro intervalos. $\mathrm{O}$ intervalo gerado pelo quarto quartil, que corresponde aos valores mais altos, acolheu os atributos considerados como pertinentes à Imagem Central. $\mathrm{O}$ terceiro quartil corresponde ao intervalo do segundo valor mais alto, configurando-se como a Primeira Imagem Intermediária. O segundo quartil corresponde ao intervalo de terceiro valor mais alto $\mathrm{e}$ foi denominado Segunda Imagem Intermediária. E, finalmente, o primeiro quartil, que corresponde ao intervalo de valores menores, denominado Periferia da Imagem (De TONI et al., 2006).

$\mathrm{Na}$ terceira, e última etapa do tratamento dos dados, foi realizada a categorização dos atributos da imagem de estratégia organizacional pelas dimensões propostas por Mintzberg (1987), os Cinco 
A percepção de gestores da construção civil acerca do conceito de estratégia organizacional: Um estudo exploratório

Ps. A análise de conteúdo também foi utilizada pelos pesquisadores, nesta etapa, com a adoção do critério do VT para ponderação dos atributos. Os resultados obtidos nesta etapa foram apresentados graficamente no Gráfico de Configuração das Imagens (GCI), o qual apresenta três informações: os atributos que compõem as imagens, a proximidade destes atributos com a Imagem Central e a dimensão ou categoria a que pertencem (um dos Cinco Ps da estratégia de Mintzberg).

\section{Análise dos resultados}

Esta seção é composta por três partes, visto que a primeira tem o objetivo de mostrar a os atributos identificados e a proximidade dos mesmos com o termo indutor; a segunda, apresenta a classificação dos atributos em relação as Dimensões de Estratégia (Cinco Ps) e, por fim, a terceira contempla o Gráfico de Configuração da Imagem (GCI).

\subsection{Identificação dos atributos e sua proximidade com o termo indutor}

Com base na análise de conteúdo das respostas obtidas com a aplicação do "Roteiro Básico de Questões", foram identificados 32 atributos. Tais atributos encontrados formam, sob o ponto de vista dos entrevistados, a imagem sobre estratégia organizacional.

Com a identificação dos atributos, e com o cálculo de VF, VO e VT, foi procedida a separação dos Quartis (vide Figura 3), o que possibilitou a apresentação, por áreas de proximidade com o termo indutor, a identificação da Imagem Central, da Primeira Imagem Intermediária, da Segunda Imagem Intermediária e da Periferia da Imagem. A Figura 3 apresenta cada um dos quartís calculados através dos intervalos de VT, deste modo a Imagem Central é composta pelos atributos que ficaram no intervalo entre 19 e 92 pontos, as imagens intermediárias que ficaram entre 7 e 18 e a periferia da imagem que foram os atributos menos frequentes e salientes.

Figura 3: Áreas de proximidade dos atributos em relação ao termo indutor

\begin{tabular}{|c|c|c|}
\hline Quartis & Proximidade com o Termo Indutor & Intervalo do VT \\
\hline 4 & Imagem Central & VT de 92 a 19 \\
3 & Primeira Imagem Intermediária & VT de 18 a 12 \\
2 & Segunda Imagem Intermediária & VT de 11 a 7 \\
1 & Periferia da Imagem & VT de 6 a 1 \\
\hline
\end{tabular}

Fonte: Dados provenientes da pesquisa.

A Figura 4 organiza os quatro níveis das imagens e apresenta como os atributos as compõem. Como pode ser observado, o conceito central de imagem de estratégia organizacional está mais fortemente direcionado a conceitos relacionados ao "planejamento", "organização", "plano de ação" e "objetivos".

Figura 4: Atributos identificados e sua proximidade com o termo indutor

\begin{tabular}{|c|c|l|c|c|c|}
\hline Imagem & $\mathbf{N}^{\mathbf{0}}$ & \multicolumn{1}{|c|}{ Atributos } & VF & VO & VT \\
\hline \multirow{2}{*}{ Imagem Central } & $\mathbf{1}$ & Planejamento & 27 & 65 & 92 \\
\cline { 2 - 7 } & $\mathbf{2}$ & Organização & 21 & 45 & 66 \\
\hline
\end{tabular}




\begin{tabular}{|c|c|c|c|c|c|}
\hline & 3 & Plano de ação, cronograma & 15 & 22 & 37 \\
\hline & 4 & Objetivos & 12 & 24 & 36 \\
\hline & 5 & Ferramenta & 9 & 23 & 32 \\
\hline & 6 & Foco & 13 & 8 & 21 \\
\hline & 7 & Direção & 8 & 13 & 21 \\
\hline & 8 & Mais rentabilidade & 10 & 10 & 20 \\
\hline \multirow{7}{*}{$\begin{array}{l}\text { Primeira Imagem } \\
\text { Intermediária }\end{array}$} & 9 & Metas & 6 & 11 & 17 \\
\hline & 10 & Melhorias organizacionais & 9 & 8 & 17 \\
\hline & 11 & Controle & 12 & 4 & 16 \\
\hline & 12 & Estratégia, como atingir objetivos & 8 & 7 & 15 \\
\hline & 13 & Caminho & 7 & 8 & 15 \\
\hline & 14 & Indispensável, necessidade, importante & 12 & 2 & 14 \\
\hline & 15 & Estrutura & 2 & 10 & 12 \\
\hline \multirow{9}{*}{$\begin{array}{l}\text { Segunda Imagem } \\
\text { Intermediária }\end{array}$} & 16 & Padronização & 10 & 1 & 11 \\
\hline & 17 & Entendimento & 6 & 5 & 11 \\
\hline & 18 & Comprometimento & 7 & 4 & 11 \\
\hline & 19 & Satisfação, realização & 11 & 0 & 11 \\
\hline & 20 & Desenvolvimento pessoal & 5 & 4 & 9 \\
\hline & 21 & Visão & 4 & 5 & 9 \\
\hline & 22 & Satisfação de clientes & 7 & 1 & 8 \\
\hline & 23 & Pontos fortes & 4 & 4 & 8 \\
\hline & 24 & Crescimento, sucesso & 7 & 0 & 7 \\
\hline \multirow{8}{*}{ Periferia da Imagem } & 25 & Pontos fracos & 4 & 1 & 5 \\
\hline & 26 & Identificação do contexto & 4 & 1 & 5 \\
\hline & 27 & Resultados & 4 & 0 & 4 \\
\hline & 28 & Marketing & 4 & 0 & 4 \\
\hline & 29 & Mais organização & 4 & 0 & 4 \\
\hline & 30 & Comportamento & 3 & 1 & 4 \\
\hline & 31 & Flexibilidade & 3 & 0 & 3 \\
\hline & 32 & Destaque & 3 & 0 & 3 \\
\hline
\end{tabular}

Fonte: Elaborada pelos autores a partir dados provenientes da pesquisa.

Nesse sentido, pode-se induzir que para os entrevistados o conceito de estratégia organizacional é um planejamento organizado ou estruturado que visa o atingimento de objetivos através de um foco de ação.

\subsection{Classificação dos atributos a partir das dimensões de estratégia (Cinco Ps)}

Através da análise de conteúdo realizado pelos pesquisadores, os atributos encontrados foram devidamente classificados dentre as cinco dimensões de estratégia organizacional sugeridas por Mintzbert (1987) (Plano, Pretexto, Padrão, Posição e Perspectiva). O resultado desta classificação é apresentado na Figura 5. 
A percepção de gestores da construção civil acerca do conceito de estratégia organizacional: Um estudo exploratório

Figura 5: Agrupamento dos atributos em suas respectivas dimensões

\begin{tabular}{|c|c|c|c|}
\hline $\begin{array}{c}\text { Dimensões } \\
\text { (Os Cinco Ps da Estratégia) }\end{array}$ & Atributos Intrínsecos à Cada Dimensão & VT & $\%$ \\
\hline \multirow{10}{*}{ Plano } & Planejamento & 92 & 16,79 \\
\hline & Plano de ação, cronograma & 37 & 6,75 \\
\hline & Objetivos & 36 & 6,57 \\
\hline & Foco & 21 & 3,83 \\
\hline & Direção & 21 & 3,83 \\
\hline & Metas & 17 & 3,10 \\
\hline & Estratégia, como atingir objetivos & 15 & 2,74 \\
\hline & Caminho & 15 & 2,74 \\
\hline & Marketing & 4 & 0,73 \\
\hline & Subtotal & 258 & 47,08 \\
\hline \multirow{5}{*}{ Pretexto } & Satisfação de clientes & 8 & 1,46 \\
\hline & Crescimento, sucesso & 7 & 1,28 \\
\hline & Flexibilidade & 3 & 0,55 \\
\hline & Destaque & 3 & 0,55 \\
\hline & Subtotal & 21 & 3,83 \\
\hline \multirow{7}{*}{ Padrão } & Ferramenta & 32 & 5,84 \\
\hline & Mais rentabilidade & 20 & 3,65 \\
\hline & Melhorias organizacionais & 17 & 3,10 \\
\hline & Padronização & 11 & 2,01 \\
\hline & Resultados & 4 & 0,73 \\
\hline & Mais organização & 4 & 0,73 \\
\hline & Subtotal & 88 & 16,06 \\
\hline \multirow{5}{*}{ Posição } & Entendimento & 11 & 2,01 \\
\hline & Visão & 9 & 1,64 \\
\hline & Identificação do contexto & 5 & 0,91 \\
\hline & Comportamento & 4 & 0,73 \\
\hline & Subtotal & 29 & 5,29 \\
\hline \multirow{10}{*}{ Perspectiva } & Organização & 66 & 12,04 \\
\hline & Controle & 16 & 2,92 \\
\hline & Indispensável, necessário, importante & 14 & 2,55 \\
\hline & Estrutura & 12 & 2,19 \\
\hline & Comprometimento & 11 & 2,01 \\
\hline & Satisfação, realização & 11 & 2,01 \\
\hline & Desenvolvimento pessoal & 9 & 1,64 \\
\hline & Pontos fortes & 8 & 1,46 \\
\hline & Pontos fracos & 5 & 0,91 \\
\hline & Subtotal & 152 & 27,74 \\
\hline \multicolumn{2}{|r|}{ Total Geral } & 548 & 100,00 \\
\hline
\end{tabular}

Fonte: Dados provenientes da pesquisa.

A Figura 5 apresenta quatro colunas: na primeira coluna (da esquerda para a direita), estão as dimensões (ou os Cinco Ps); na segunda, os atributos vinculados a cada dimensão; na terceira, o Valor Total (VT), que é resultante da soma do Valor de Frequência (VF) com o Valor de Ordem (VO); e, na quarta e última coluna, os percentuais relativos ao VT. Além disso, ao final de cada dimensão (ou ao final de cada um dos Ps), é apresentado o subtotal, tanto em pontuação (VT), quanto em percentual. 
Os resultados apontam que as dimensões que concentram a maior parte dos 32 atributos identificados são as seguintes dimensões: Plano, com 47,08\% e Perspectiva, com $27,74 \%$ dos Valores Totais (VTs) dos atributos. Sendo assim, é possível concluir que, para os entrevistados, o planejamento estratégico está fortemente relacionado a Plano e Perspectiva. Os dois atributos que atingiram os maiores VTs foram "Planejamento", com 16,79\%" e "Organização", com 12,04\%; atributos estes que estão localizados, respectivamente, nas duas dimensões de maior VT encontrados na pesquisa realizada. Dentre as outras três dimensões, a dimensão Padrão apresenta 16,06\%; Posição, 5,29\% e, por fim, Pretexto, com apenas 3,83\% dos VTs.

No que se refere às diretrizes que compõem cada uma das cinco dimensões, pode ser dito que, para a dimensão Plano, os dois principais atributos são "Planejamento" (16,79\%) e "Plano de ação, cronograma" (6,75\%). Quanto à dimensão Pretexto, os atributos
"Satisfação de clientes" (1,46\%) e "Crescimento, sucesso" (1,28\%) foram os de maior peso. A dimensão Padrão, por sua vez, apresenta os atributos "Ferramenta" (5,84\%) e "Mais rentabilidade" (3,65\%) como os mais salientes; enquanto que, na dimensão Posição, destacaram-se os atributos "Entendimento" (2,01\%) e "Visão" $(1,64 \%)$. Por fim, na dimensão Perspectiva, os atributos de maior peso foram "Organização" (12,04\%) e "Controle" $(2,92 \%)$.

\subsection{Gráfico de configuração da imagem (GCI)}

A Figura 6 apresenta o Gráfico de Configuração da Imagem (GCI), que mostra as principais informações relacionadas à imagem resultante a respeito do conceito de Estratégia Organizacional, fruto da percepção dos gestores entrevistados. Para facilitar a interpretação do GCI, segue, a Figura 7, na qual consta a lista dos 32 atributos.

Figura 6: GCI representa o conceito de Estratégia Organizacional

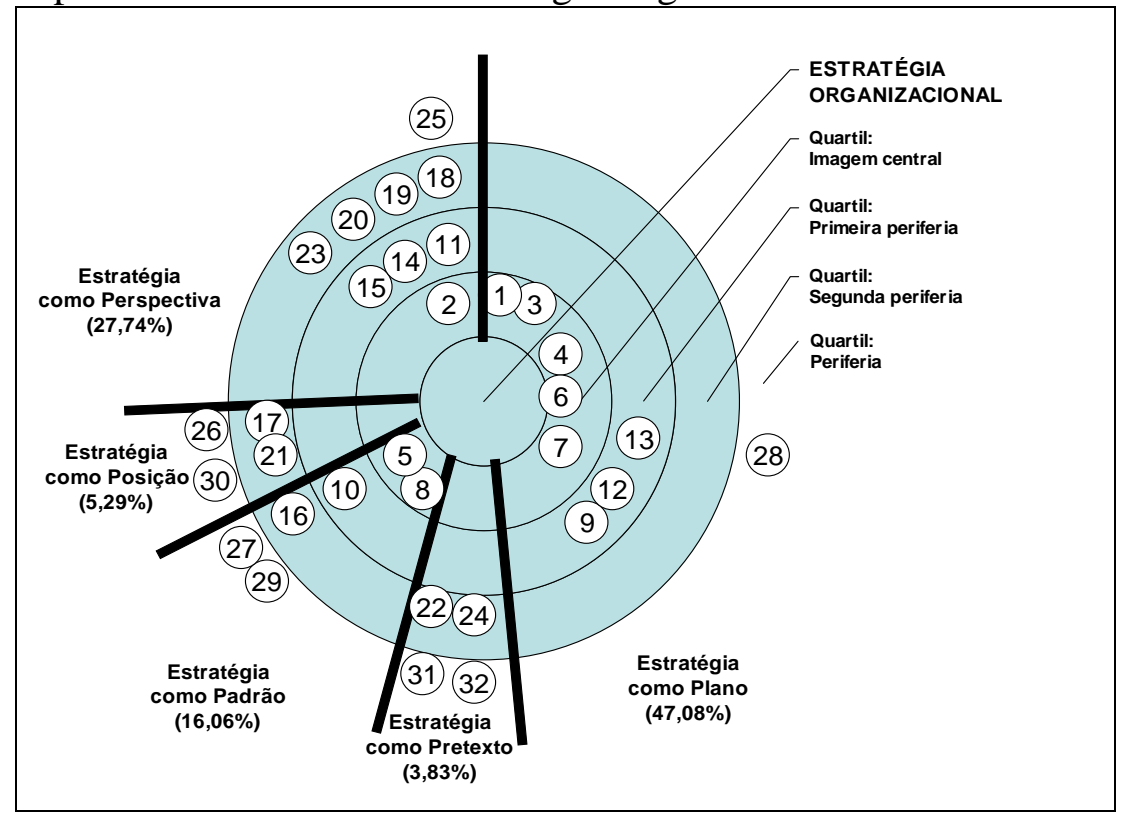

Fonte: Elaborado pelos autores a partir dados provenientes da pesquisa. 
A percepção de gestores da construção civil acerca do conceito de estratégia organizacional: Um estudo exploratório

É oportuno comentar que o GCI disponibiliza três informações úteis para a visualização da imagem (De TONI et al., 2006) em estudo: (i) os atributos que compõem a imagem acerca da Estratégia
Organizacional; (ii) as categorias ou dimensões de atributos, a partir dos Cinco Ps da estratégia, propostos por Mintzberg (1987); e (iii) a proximidade dos atributos em relação ao termo indutor empregado (Estratégia Organizacional).

Figura 7: Lista de atributos (os quais compõem o GCI)

\begin{tabular}{|r|l|l|l|}
\hline$N^{\mathbf{0}}$ & \multicolumn{1}{|c|}{ Atributos } & $\mathbf{N}^{\mathbf{0}}$ & \multicolumn{1}{c|}{ Atributos } \\
\hline $\mathbf{1}$ & Planejamento & $\mathbf{1 7}$ & Entendimento \\
\hline $\mathbf{2}$ & Organização & $\mathbf{1 8}$ & Comprometimento \\
\hline $\mathbf{3}$ & Plano de ação, cronograma & $\mathbf{1 9}$ & Satisfação, realização \\
\hline $\mathbf{4}$ & Objetivos & $\mathbf{2 0}$ & Desenvolvimento pessoal \\
\hline $\mathbf{5}$ & Ferramenta & $\mathbf{2 1}$ & Visão \\
\hline $\mathbf{6}$ & Foco & $\mathbf{2 2}$ & Satisfação de clientes \\
\hline $\mathbf{7}$ & Direção & $\mathbf{2 3}$ & Pontos fortes \\
\hline $\mathbf{8}$ & Mais rentabilidade & $\mathbf{2 4}$ & Crescimento, sucesso \\
\hline $\mathbf{9}$ & Metas & $\mathbf{2 5}$ & Pontos fracos \\
\hline $\mathbf{1 0}$ & Melhorias organizacionais & $\mathbf{2 6}$ & Identificação do contexto \\
\hline $\mathbf{1 1}$ & Controle & $\mathbf{2 7}$ & Resultados \\
\hline $\mathbf{1 2}$ & Estratégia, como atingir objetivos & $\mathbf{2 8}$ & Marketing \\
\hline $\mathbf{1 3}$ & Caminho & $\mathbf{2 9}$ & Mais organização \\
\hline $\mathbf{1 4}$ & Indispensável, necessidade, importante & $\mathbf{3 0}$ & Comportamento \\
\hline $\mathbf{1 5}$ & Estrutura & $\mathbf{3 1}$ & Flexibilidade \\
\hline $\mathbf{1 6}$ & Padronização & $\mathbf{3 2}$ & Destaque \\
\hline
\end{tabular}

Fonte: Dados provenientes da pesquisa.

Com base no GCI de Estratégia Organizacional, verifica-se que a Imagem Central é composta por oito atributos (planejamento, organização, plano de ação/ cronograma, objetivos, ferramenta, foco, direção e mais rentabilidade). Dentre os Cinco Ps de Mintzberg, cinco dentre os oito atributos indicados estão localizados na dimensão Plano, sendo que os demais são relativos, respectivamente, às dimensões Padrão e Perspectiva. A dimensão de menor saliência foi a de Pretexto; próxima a esta, a estratégia vista como Posição também teve uma baixa indicação. Ambas, com dois atributos na Segunda Imagem Periférica e outros dois atributos na Periferia da Imagem.
Portanto, a estratégia sob a perspectiva de um Plano foi a dimensão que mais se aproxima da Imagem Central (ou Núcleo central) de Estratégia Organizacional. Com base nesta informação, é possível supor que os gestores entrevistados e, consequentemente, as organizações onde atuam, em posição de tomadores de decisão, buscarão conduzi-las por meio de planejamentos estratégicos adotados em direção às questões (ou aos atributos) componentes, inerentes a esta dimensão da estratégia.

Como observado em parágrafo anterior, a dimensão de menor indicação foi a da estratégia com um Pretexto, com 
somente $3,83 \%$ do VT encontrado no conjunto geral de atributos da imagem. Tal informação, seria cabível, e válida, na tentativa de ressaltar, junto aos gestores alvos deste estudo, a importância desta dimensão para o desenvolvimento de suas organizações. Relevante observar, que o atributo de maior VT diz respeito ao "Planejamento", componente da estratégia como Plano. Já o atributo de menor VT, "Flexibilidade", juntamente com "Destaque" ocupa o quartil da Periferia da Imagem, atinente à dimensão Pretexto, que, por sua vez, é a dimensão de menor saliência. Tal observação permite que os profissionais (gestores, tomadores de decisões), ao desenvolverem o planejamento estratégico de suas organizações percebam a importância e deem atenção necessária a esta dimensão.

\section{Considerações Finais}

As imagens, enquanto representações, são provavelmente $\mathrm{o}$ principal conteúdo do pensamento humano. Elas constituem um dos materiais intelectuais mais importantes do homem, sendo capazes de influenciar e direcionar o comportamento das pessoas. Neste sentido compreender como os gestores formam a imagem de seus conceitos constitui um importante trunfo para melhorar o porquê algumas organizações obtêm desempenhos tão diferentes. Dentre as contribuições deste artigo três dimensões merecem destaque. A primeira contribuição refere-se à proposição do método para identificar as imagens. Para tanto foi empregada a Técnica de Configuração de Imagens Ideativas (TCID). Ao se considerar os resultados obtidos através da utilização desta técnica para compreender como uma imagem pode ser configurada na mente dos entrevistados. Entender o conceito a partir da imagem central e periférica é possível identificar quais atributos são mais frequentes e relevantes para os entrevistados. De posse destes atributos é possível identificar quais imagens direciona o comportamento das pessoas. Uma segunda contribuição deste artigo esta na identificação do conceito de estratégia sob a ótica dos Cinco Ps definidos por Mintzberg (1987). Os achados mostram que para os entrevistados o conceito de estratégia organizacional está mais direcionado a definição de um Plano e Perspectiva, o que mostra que os demais conceitos ainda não estão presentes de forma mais intensa. Uma terceira contribuição desta pesquisa constitui na identificação do conceito geral de estratégia organizacional. Assim, para os entrevistados o conceito de estratégia organizacional é um planejamento organizado, estruturado que visa o atingimento de objetivos através de um foco de ação.

Os achados deste estudo são importantes para melhor entender a forma de pensar dos gestores e como estas formas podem direcionar suas ações. Através do Gráfico de Configuração da Imagem (GFI) é possível identificar de forma resumida e didática a estrutura conceitual de estratégia organizacional para os entrevistados. Assim, as três importantes informações: conjunto de atributos formadores do conceito, sua proximidade com o núcleo central e as categorias na qual pertencem o conceito, auxiliam à mapear o modelo mental dos entrevistados com relação ao objeto de estudo. Assim, tais achados podem auxiliar a mapear outros tipos de conceitos e modelos mentais do público alvo para primeiro compreender melhor os conceitos explorados e posteriormente direcionar ações que auxiliem a manter, ampliar ou modificar os conceitos existentes.

Dentre as limitações desta pesquisa pode-se destacar primeiro o fato de ser uma pesquisa exploratória em que trouxe uma proposição teórica e uma metodologia identificada com apenas 20 empreendedores, num ambiente específico, a construção civil. Novos estudos podem 
A percepção de gestores da construção civil acerca do conceito de estratégia organizacional: Um estudo exploratório

ser feitos no sentido de ampliar a amostra e em diferentes setores com o propósito de fazer comparativos entre as imagens identificadas. Uma segunda limitação pode estar na categorização dos atributos a partir dos 5 Ps de estratégia. Tal categorização pode ter limitações, uma vez que um conceito carrega consigo inúmeros significados que dentro dele pode ter elementos tanto de plano quanto de pretexto e outros. Dessa forma, futuras pesquisas podem ser direcionadas no sentido de buscar através da semiótica, por exemplo, identificar o real sentido do atributo apresentado. Uma outra pesquisa também pode ser encaminhada para compreender se os entrevistados ou diferentes pesquisadores conseguem identificar a partir dos atributos listados se eles se enquadram ou não nas categorias propostas.

De qualquer forma, este estudo é importante uma vez que a identificação das imagens de conceitos, como o conceito de estratégia organizacional, constitui uma excelente oportunidade para melhor compreender determinados comportamentos no ambiente empresarial, de modo a possibilitar a implementação de ações para manter ou modificar a imagem existente e seus respectivos efeitos e resultados.

\section{Referências}

ABRIC, J. C. A theoretical and experimental approach to the study of social representations in a situation of interaction. In: FARR.; R. M.; MOSCOVICI, S. (Eds.) Social representations. Cambridge: Cambridge University Press, p. 169-183, 1984.

ABRIC, J. C. Specific processes of social representations. Papers on Social Representations. Linz, v. 5, p. 77-80, 1996.

AKHTER, S. H.; ANDREWS, J. C.; DURVASULA, S. The influence of retail store environment on brand-related judgments. Journal of Retailing and Consumer Service, v. 1, n. 2, p. 67-76, 1994.

APDG - Assessoria de Pesquisa e Desenvolvimento Gerencial. Customer Window: catálogo de informações. São Paulo: Rhodia, 1994.

BARDIN, L. Análise de conteúdo. 1. ed. Lisboa: Edições 70, 2011.

BARICH, H.; KOTLER, P. A framework for marketing image management. Sloan Management Review, v. 32, n. 2, p. 97104, 1991.

BREAKWELL, G. M. Mental models and social representations of hazards: the significance of identity processes. Journal of Risk Research, v. 4, n. 4, p. 341-351, 2001.

BOULDING, K. E. The image: knowledge in life and society. 6. Ed. Ann Harbor: University of Michigan, 1968.

CHAPMAN, J. A.; FERFOLJA, T. Fatal flaws: the acquisition of imperfect mental models and their use in hazardous situations. Journal of Intellectual Capital, v. 2, n. 4, p. 398-409, 2001.

DAFT, R. L.; Organization Theory \& design. 11. ed. South-Western: Cengage Learning, 2012.

De TONI, D. Administração da imagem de produtos: desenvolvendo um instrumento para a configuração da imagem de produto. Tese (Doutorado em Administração). Escola de Administração, Universidade Federal do Rio Grande do Sul, Programa de Pós-Graduação em Administração. Porto Alegre, 2005.

De TONI, D.; MILAN, G. S.; SCHULER, M. Configuração de imagens de serviços: um estudo aplicado aos serviços de fisioterapia disponibilizados por um plano de saúde. In: Encontro Anual da ANPAD, 29., 2005, Brasília. Anais... Brasília: ANPAD, 2005. 
De TONI, D.; MILAN, G. S.; SCHULER, M.; LARENTIS, F.; HEXSEL, A. E. A imagem como fator da inovação nas empresas: um estudo exploratório a partir da configuração das imagens dos gestores sobre estratégia organizacional. In: XXIV Simpósio de Gestão da Inovação Tecnológica. Anais... Gramado: ANPAD, 2006.

DICHTER, E. What's in an image. Journal of Consumer Marketing, v. 2, $\mathrm{n}$. 1, p. 75-81, 1985.

EISENHARDT, K. M. Strategy as strategic decision making. Sloan Management Review, v. 40, n. 3, p. 6572, 1999.

GENTNER, D. Psychology of mental model. In: SMELSE, N.; BATES, P. (Eds.). International Encyclopedia of the Social and Behavioral Science. London: Elsevier, 2001.

IBGE - Instituto Brasileiro de Geografia e Estatística. Site institucional. Disponível em:

http://www.ibge.gov.br/censo2010/dados_ divulgados/index.php?uf=43. Acesso em: 08 dez. 2010.

MACHADO-DA-SILVA, C. L.; FONSECA, V. S.; FERNANDES, B. H. R. Cognição e institucionalização na dinâmica da mudança em organizações. In: RODRIGUES, S. B.; CUNHA, M. P. (org.) Estudos organizacionais: novas perspectivas na administração de empresas, uma coletânea luso-brasileira. São Paulo: Iglu, p. 123-150, 2000.

MALHOTRA, N. K. Marketing Research - an applied orientation. 6. ed. Porto Alegre: Prentice Hall, 2009.

MILAN. G. S.; De TONI, D. A configuração das imagens dos gestores sobre o conceito de estratégia. Revista de Administração da Mackenzie, v. 9, n. 6, p. 102-125, 2008.
MINAYO, M. O conceito de representações sociais dentro da sociologia clássica. In: JOVCHELOVITCH, S.; GUARESCHI, P. (org.) Textos em representações sociais. Petrópolis: Vozes, 1997.

MINTZBERG, H. The strategy concept I: five Ps for strategy. California Management Review, v. 30, n. 1, p. 1124, 1987.

MINTZBERG, H. Ascensão e queda do planejamento estratégico. Porto Alegre: Bookman, 2004.

MINTZBERG, H.; LAMPEL, J.; QUINN, J. B.; GHOSHAL, S. O processo da estratégia: conceitos, contextos e casos selecionados. 4. ed. Porto Alegre: Bookman, 2006.

MOSCOVICI, S. A representação social da psicanálise. Rio de Janeiro: Zahar, 1978.

MOSCOVICI, S. Notes towards a description of social representations. European Journal of Social Psychology, v. 18, p. 211-250, 1988.

MOSCOVICI, S. Social representation: explorations in social psychology. Cambridge: Polity Press, 2000.

NEVES, R. C. Imagem empresarial: como as organizações [e as pessoas] podem proteger e tirar partido do seu maior patrimônio. 3. ed. Rio de Janeiro: Mauad, 2003.

OLIVEIRA, M. K. Vygotsky: aprendizado e desenvolvimento um processo sóciohistórico. 3. ed. São Paulo: Scipione, 1995.

PORTER, M. E. What is strategy? Harvard Business Review, v. 74, n. 6, p. 61-78, 1996.

PORTER, M. E. Estratégia. In: Expo Management. São Paulo: HSM Management, 2001. 
A percepção de gestores da construção civil acerca do conceito de estratégia organizacional: Um estudo exploratório

PORTER, M. E. Competitive advantage:

Creating and sustaining superior performance. New York: The Free Press, 2004.

QUINN, J. B. Strategies for change: logical incrementalism. Homewood: Irwin, 1980.

RIBEIRO, J. L. D.; MILAN, G. S. Planejando e conduzindo entrevistas individuais. In: RIBEIRO, J. L. D.; MILAN, G. S. (eds.). Entrevistas individuais: teoria e aplicações. Porto Alegre: FEEng/UFRGS, 2004. cap. 1, p. 922.

SÁ, C. P. Sobre o núcleo central das representações sociais. Petrópolis, RJ: Vozes, 1996.

SÁ, C. P. Núcleo central das representações sociais. 2.ed. rev. Petrópolis, RJ: Vozes, 2002.

SCHULER, M. Management of the organizational image: a method for organizational image configuration. Corporate Reputation Review, v. 7, n. 1, p. 37-53, Spring 2004.

SCHULER, M.; DE TONI, D.; MILAN, G. S. New developments in ICM: Image configuration method. African Journal of Management, v.8, n.4, p. 180-190, 2014.

SENGE, P. A quinta disciplina. 28. ed. Rio de Janeiro: Best Seller, 2012.

THOMPSON JR., A. A.; STRICKLAND III, A. J. Planejamento estratégico: elaboração, implementação e execução. São Paulo: Thomson Pioneira, 2004.

VERGÈS, P. L'evocation de lárgent: une méthode pour la definition du noyau central dune presentation. Bulletin de Psychologie, v. 45, n. 405, p. 203-209, 1992.

ZALTMAN, G. Rethinking market research: putting people back. Journal of Marketing Research, v. 34, n. 4, p. 456472, 1997. 